\title{
Preface Issue 4-2015
}

\author{
Hans-Christoph Grunau ${ }^{1}$
}

(C) Deutsche Mathematiker-Vereinigung and Springer-Verlag Berlin Heidelberg 2015

In their survey article on "Stochastic PDEs and lack of regularity", Dirk Blömker and Marco Romito discuss a nonlinear partial differential equation which may serve as a phenomenological model for the growth of an amorphous surface. This equation contains an additional noise term which models fluctuations of the flow of incoming particles. The authors mention that similar equations are used to model the formation of sand or snow ripples and that their model has striking similarities to the KadarParisi-Zhang (KPZ) equation. While it is possible to prove local (in time) existence of strong solutions, which are also unique, the problem of whether they are global (in time) or may blow up in finite time remains an important open problem. In one space dimension (i.e., assuming invariance with respect to the second spatial variable) the authors prove the existence of global (in time) weak solutions where, however, uniqueness is left open. Similar difficulties due to the lack of regularity arise also e.g. in the time-dependent 3D-Navier-Stokes equations.

Jacques Tits is one of the most influential group theorist and algebraic geometers and received, among many other awards, in 1996 the Cantor medal of the Deutsche Mathematiker-Vereinigung and in 2008 the Abel Prize of the Norwegian Academy of Science and Letters. Two years ago, Jacques Tits' "Collected Works" appeared which are subject of this issue's "Classics Revisited"-contribution. Linus Kramer highlights some of their contents grouping them according to their themes.

Walter D. van Suijlekom gives a summary and his opinion of Frederic Paugam's recent book "Towards the mathematics of quantum field theory".

\footnotetext{
H.-Ch. Grunau

hans-christoph.grunau@ovgu.de

1 Institut für Analysis und Numerik, Fakultät für Mathematik, Otto-von-Guericke-Universität, Postfach 4120, 39016 Magdeburg, Germany
} 